\title{
Anesthetic Management of a Patient with Polycythemia Vera for Nephrectomy
}

Sethi S*, Kumari K

$$
\text { Case Report }
$$

Department of Anaesthesia and Intensive Care, Post Graduate Institute of Medical Education and Research (PGIMER), Chandigarh, India.

\section{Abstract}

Polycythemia Vera (PV) is a chronic myeloproliferative disease, characterized by erythrocytosis and hyperviscosity. A high proportion of PV surgeries are complicated by thrombosis $(7.7 \%)$ or by a major hemorrhage $(7.3 \%)$, increasing morbidity and mortality among these patients. We report a 28 -year-old male patient with PV and renal cell carcinoma posted for nephrectomy. He was found to have a hematocrit of $68 \%$ on routine work-up. Preoperatively he was phlebotomized 4 times to decrease hematocrit to $48 \%$. Perianesthetic considerations and anesthetic management of this patient are discussed. Pretreatment of PV with phlebotomy, adequate hydration, and recognition of thrombotic and bleeding problems decreases perioperative morbidity and mortality and are the keystones in the successful anesthetic management of such patients.

Keywords: Polycythemia Vera; Nephrectomy; Phlebotomy; Anesthetic; Management.

\section{*Corresponding Author:}

Dr. Sameer Sethi,

Assistant Professor, Department of Anaesthesia and Intensive Care, Post graduate Institute of Medical Education and Research, Sector 12, Chandigarh, India.

Tel: 0172-2756501/07087008491

E-mail: sethi.sameer@rediffmail.com

Received: January 25, 2015

Accepted: August 11, 2015

Published: August 14, 2015

Citation: Sethi S, Kumari K(2015) Anesthetic Management of a Patient with Polycythemia Vera for Nephrectomy. Int J Anesth Res. 3(7), 147-149. doi: http://dx.doi.org/10.19070/2332-2780-1500036

Copyright: Sethi $\mathbf{S}^{\odot}$ 2015. This is an open-access article distributed under the terms of the Creative Commons Attribution License, which permits unrestricted use, distribution and reproduction in any medium, provided the original author and source are credited.

\section{Introduction}

Polycythemia Vera (PV) is a hematopoietic stem cell disorder characterized by erythrocytosis and hyperviscosity [1]. Hyperviscosity causes blood stasis and increases the risk of arterial and venous thrombosis [2]. PV is also associated with platelet dysfunction, increasing the risk of paradoxical hemorrhage in the perioperative period [3]. A high proportion of PV surgeries are complicated by thrombotic events (myocardial infarction, pulmonary embolism, cerebrovascular accident) and bleeding diathesis $[2,3]$. The cumulative rate of thrombosis ranges from 2.5 to $5.0 \%$ per patient-year in PV [4]. Phlebotomy and avoidance of extreme dehydration in these patients lower the risk of both thrombosis and hemorrhage in the perioperative period. Perianesthetic considerations and anesthetic management of a 28-year-old man, diagnosed to have PV on routine work-up and posted for nephrectomy for renal cell carcinoma under general anesthesia is discussed.

\section{Case Report}

28-year-old male having renal cell carcinoma was admitted to our hospital for nephrectomy. Routine hematologic investigations revealed a hemoglobin level of $22.3 \mathrm{~g} / \mathrm{dL}$, hematocrit of $67.9 \%$, total white blood cell count of $7800 / \mu \mathrm{L}$, and platelet count of $100 \mathrm{x}$ $10^{3} / \mu \mathrm{l}$. To rule out laboratory errors, hemoglobin and hematocrit were repeated from another laboratory, that showed similar results. Serum erythropoietin level was 25.2, which was in normal range. Arterial blood gas analysis showed normal oxygenation [arterial partial pressure of oxygen $\left(\mathrm{PaO}_{2}\right)$ of 95.6, oxygen saturation $\left(\mathrm{SPO}_{2}\right)$ of $97.4 \%$ ] and a hematocrit of $73 \%$. Based on the reports of hemogram, erythropoietin and arterial blood gas analysis, the diagnosis of PV was confirmed. The patient did not give any history of symptoms suggestive of the condition. His coagulation profile, electrolytes, liver and renal function tests and chest Xray were normal. Electrocardiogram (ECG) showed left ventricle hypertrophy for which transthoracic echocardiogram was done which was normal. Patient had a past medical history of hypertension from last 2 years for which he was taking the tablet (tab) telmisartan 40/12.5 mg, tab amlong $10 \mathrm{mg}$, tab metoprolol 50mg and his blood pressure was controlled with these medications. For increased hematocrit, hematological consultation was done, who advised phlebotomy to decrease hemetocrit. The patient underwent phlebotomy four times and each time $350 \mathrm{ml}$ of blood was removed. After fourth phlebotomy, his hemoglobin and hematocrit decreased to $17.6 \mathrm{~g} / \mathrm{dl}$ and $48 \%$ respectively. One day prior to surgery, he was again evaluated and was advised premedication with oral ranitidine $150 \mathrm{mg}$ and alprazolam $0.25 \mathrm{mg}$ on the previous night and on the morning of surgery, to decrease the risk of aspiration and anxiety. Tab telmisartan was withheld on the day of surgery and other antihypertensives were continued. Adequate hydration and hemodilution were achieved with intravenous infusion of Ringer's lactate solution at $2 \mathrm{ml} / \mathrm{kg} / \mathrm{hr}$ during period of fasting. General anesthesia with epidural was planned. Intravenous (i.v) access was secured with two peripheral i.v cannula 
(18 and 16 gauge). Standard non-invasive anesthesia monitoring (ECG, noninvasive blood pressure, pulse oximetry, capnography, temperature) was done. For continuous invasive blood pressure monitoring left radial artery was cannulated. For intraoperative and postoperative analgesia, epidural catheter was inserted in L1 - L2 interspinous space. Anesthesia was induced with propofol $100 \mathrm{mg}$, fentanyl $100 \mu \mathrm{g}$, atracurium $25 \mathrm{mg}$ and maintained with nitrous oxide in oxygen $50 \%$ and isoflurane with a cumulative MAC of $1-1.2 \%$. The patient's vitals remained stable throughout the procedure. Blood loss was minimal which was replaced with Ringer's lactate solution. After extubation in the immediate postoperative period patient was shifted to the high dependency unit (HDU) for monitoring for 24 hours. Postoperative analgesia was ensured with continuous administration of $0.125 \%$ bupivacaine at $5 \mathrm{ml} / \mathrm{hr}$ via the epidural catheter during the first $48 \mathrm{~h}$. Thereafter, the catheter was removed. Postoperatively patient was monitored in the ward for five days and then discharged home in stable condition.

\section{Discussion}

First described in 1892, PV is not a new disease with an incidence of 0.7-2.6 per 100000 individuals [5]. The incidence of $\mathrm{PV}$ increases with age although it can be seen in all age groups [5, 6]. Polycythemia is mainly classified into primary and secondary polycythemia. Primary polycythemia (polycythemia Vera) is a disorder of the blood-producing cells of the bone marrow that results in increased production of red blood cells resulting in erythrocytosis and increased hematocrit [1]. Increased hematocrit leads to hyperviscosity and blood stasis which in turn predisposes the cardiovascular and central nervous system to thrombotic events [2]. Pathological hemorrhages are also common, especially during or after surgery due to impaired platelet function [3]. Secondary polycythemia is caused by hypoxia, smoking, high altitude, chronic pulmonary and cyanotic heart disease [7]. The criteria for a diagnosis of PV include an increased hematocrit, normal erythropoietin levels, normal arterial oxygenation, and splenomegaly not due to another cause [8]. Our patient had primary polycythemia with a hematocrit of $68 \%$, splenomegaly and normal erythropoietin level and arterial oxygenation.

A high proportion of PV surgeries are complicated by thrombosis or by a major hemorrhage, contributing to the main reasons for both morbidity and mortality among these patients [9]. The cumulative rate of thrombosis ranges from 2.5 to $5.0 \%$ per patientyear in PV [4]. Ruggeri M, et al in their retrospective survey concluded that a high proportion of PV surgeries were complicated by vascular thrombosis $(7.7 \%)$ or by a major hemorrhage $(7.3 \%)$ [10]. The thrombosis can occur in both the arterial and/or venous circulation and contributes to $45 \%$ of death in this population [4]. Various treatment modalities in form of phlebotomy and myelosuppressive agents such as hydroxyurea, alpha interferon, chlorambucil, busulfan, etc. are available for PV [1, 8]. Myelosuppressive drugs can reduce the rate of thrombosis, but their use raises the risk of transformation into acute leukemia. To avoid this risk, myelosuppressive drugs are indicated in only high-risk patients of more than 60 years of age or those with a past history of any thrombotic event. The drug of choice is hydroxyurea because of its efficacy in preventing thrombosis and low leukemogenicity. In Low risk patients, below 60 years of age and without any additional risk factor for thrombotic event, phlebotomy and lowdose aspirin is usually the treatment of choice with the goal of aiming the hematocrit level to less than 45 percent $(0.45)$ or even lower $[8,11]$. Wasserman and Gilbert have shown in their study that optimization and treatment of patients with uncontrolled polycythemia preoperatively, decreases peri-operative morbidity and mortality [3]. Studies have shown that oxygen delivery and oxygenation of the brain improves when hematocrit is reduced below 45\% [12]. Our patient was 28 years young male, who had no past history of thrombosis, but had a very high hematocrit of $68 \%$ and on a hematologist consultation phlebotomy was advised prior to surgery. So he underwent phlebotomy four times before surgery to reduce the hematocrit to $48 \%$.

Because of the risk pathological hemorrhage due to impaired platelet function, neuraxial blocks should advocated in those patients only, in which the platelet function and coagulation profile are normal. Our patient had a normal platelet count and normal coagulation profile and so epidural catheter was inserted for post operative analgesia. Case reports are available which demonstrates that in PV patients with normal coagulation, both general anesthesia $[13,14]$ and neuraxial techniques $[15,16]$ can be safely used. We emphasize the need of a perioperative optimization, careful prior anesthetic planning in expert hands to decrease perioperative morbidity and mortality in P.V patients.

\section{Conclusion}

PV is associated with high perioperative morbidity and mortality if complications in form of thrombosis and hemorrhage occur. Perioperative optimization of a patient with phlebotomy, adequate hydration, and recognition of thrombotic and bleeding problems decreases perioperative morbidity and mortality and are the keystones in the successful anesthetic management of such patients.

\section{References}

[1]. Beutler E (2001) Polycythemia. In Williams Haematology. (6th edtn), McGrawHill, New York. 689-701.

[2]. Reikvam H, Tiu RV (2012) Venous thromboembolism in patients with essential thrombocythemia and polycythemia vera. Leukemia 26(4): 563571.

[3]. Wasserman LR, Gilbert HS (1964) Surgical bleeding in polycythemia vera. Ann New York Acad Sci 115: 122-138.

[4]. Marchioli R, Finazzi G, Landolfi R, Kutti J, Gisslinger H, et al. (2005) Vascular and neoplastic risk in a large cohort of patients with polycythemia vera. J Clin Oncol 23(10): 2224-2232.

[5]. Johansson P (2006) Epidemiology of the myeloproliferative disorders polycythemia vera and essential thrombocythemia. SeminThromb Hemost 32(3): 171-173.

[6]. Tefferi A (2003) Polycythemia vera: a comprehensive review and clinical recommendations. Mayo Clin Proc 78(2): 174-194.

[7]. Kjeldsberg CR (2000) Polycythemia: primary and secondary. Practical diagnosis of hematologic disorders. (3rd edtn), ASCP Press, Chicago. 121.

[8]. Berlin NI (2002) Polycythemia vera: diagnosis and treatment 2002. Expert Rev Anticancer Ther 2(3): 330-336.

[9]. Barbui T, Barosi G, Birgegard G, Cervantes F, Finazzi G, et al. (2011) Philadelphia-negative classical myeloproliferative neoplasms: critical concepts and management recommendations from European LeukemiaNet. J Clin Oncol 29(6): 761-770.

[10]. Ruggeri M, Rodeghiero F, Tosetto A, Castaman G, Scognamiglio F, et al. (2008) Postsurgery outcomes in patients with polycythemia vera and essential thrombocythemia: a retrospective survey. Blood 111(2): 666-671.

[11]. Finazzi G, Barbui T (2007) How I treat patients with polycythemia vera. Blood 109(12): 5104-5111.

[12]. Thomas DJ, Marshall J, Russell RW, Wetherley-Mein G, du Boulay GH et al. (1977) Effect of hematocrit on cerebral blood flow in man. Lancet 2(8045): 941-943.

[13]. Ahmed A, Shah KA (2004) Peri-operative management of a patient with uncontrolled Polycythemia Vera for above knee amputation. J Pak Med Assoc 54(1): 34-36. 
[14]. Bouchikhi AA, Tazi MF, Mellas S, Amiroune D, Elammari JE, et al. (2013) Polycythemia vera revealed via a bladder tumor in a patient with erectile dysfunction: a case report. J Med Case Rep 7: 85.

[15]. Schmitt HJ, Becke K, Neidhardt B (2001) Epidural anesthesia for cesarean delivery in a patient with polycythemia rubra vera and preeclampsia. Anesth
Analg 92(6): 1535-1537.

[16]. Kamat S, Deepa C, Priolkar S, Nazareth M (2012) A case of polycythemia vera for orthopedic surgery: Perianesthetic considerations. Saudi J Anaesth 6(1): $87-88$. 